\title{
Progress of the Development of the IPP RF Negative Ion Source for the ITER Neutral Beam System
}

\author{
P. Franzen, H. D. Falter, U. Fantz, W. Kraus, M. Berger, S. Christ, M. Fröschle, \\ R. Gutser, B. Heinemann, S. Hilbert, S. Leyer, A. Lümkemann, C. Martens, \\ P. McNeely, R. Riedl, E. Speth, D. Wünderlich
}

\author{
Max-Planck-Institut für Plasmaphysik, EURATOM Association \\ PO Box 1533, D-85740 Garching, Germany
}

e-mail contact of main author: peter.franzen@ipp.mpg.de

\begin{abstract}
IPP Garching has successfully developed a RF driven negative ion source for the ITER neutral beam injection system. The RF source is now an interesting alternative to the reference design with filamented sources due to its in principle maintenance-free operation. Current densities of $330 \mathrm{~A} / \mathrm{m}^{2}$ and $230 \mathrm{~A} / \mathrm{m}^{2}$ have been achieved for hydrogen and deuterium, respectively, at a pressure of $0.3 \mathrm{~Pa}$ and an electron/ion ratio of 1 for a small extraction area $\left(7.0 \times 10^{-3} \mathrm{~m}^{2}\right)$ and short pulses $(<4 \mathrm{~s})$. Reliable deuterium operation with more than 150 pulses in the required parameter range was obtained by an improved cesium operation utilizing a control of all source temperatures (grid as well as source body) and monitoring the amount of cesium in the source. The development concentrates now on extending the pulse length to up to 1 hour and extending the size of the source. The long pulse test bed went into operation last year; pulses of up to some 100 seconds with more or less stable conditions in terms of extracted currents and Cs dynamics have been achieved with current densities in the range of $150-200 \mathrm{~A} / \mathrm{m}^{2}$ in hydrogen operation. The pulse length, however, is still limited by non-sufficient cooling of some parts of the source and the RF circuit. The commissioning of the so-called "half-size" source test facility started recently with the first plasma pulses; this large RF source with the width and half the height of the ITER NNBI source is dedicated to the demonstration of the homogeneity of a large RF plasma - extraction is foreseen in a latter phase — and the tests of an ITER-relevant RF circuit.
\end{abstract}

PACS: 28.52.Cx, 29.25.Ni, 52.50.Dg, 52.50.Gj

\section{Introduction}

The ITER neutral beam heating and current drive system [1] is based on the acceleration of negative hydrogen ions due to their high neutralization efficiency (0.6) at the required $1 \mathrm{MeV}$ beam energy (see table 1). In order to inject the required $17 \mathrm{MW}$, the source has to deliver $40 \mathrm{~A}$ of negative ion current. As alternative to the filamented arc sources being presently designed in the ITER neutral beam system, IPP Garching is currently developing a RF source for negative hydrogen ion production $[2,3]$. RF sources for the production of positive hydrogen ions [4-6] were developed at IPP for the neutral beam heating systems of the fusion experiments ASDEX Upgrade and W7-AS and have been successfully operational during the last decade.

Compared to arc sources RF sources are basically maintenance-free in operation. The arc source filaments have a limited lifetime requiring a remotely handled replacement few times in a year in the case of ITER. Furthermore RF sources are cheaper to build as they have fewer parts, requiring just a source body, a RF coil and a matching circuit. In addition there may be an advantage with respect to cesium consumption, which is needed to enhance the source performance (see below), as in the RF source there is no filament material that is evaporated and buries the Cs layer on the walls [7]. Present large filamented negative ion sources suffer from significant plasma non-uniformities [8,9] which are assumed to be caused by the magnetic filter field. 
Table 1: ITER neutral beam system: requirements and achievements of the IPP NNBI ion source at the test facilities BATMAN and MANITU.

\begin{tabular}{lccc}
\hline Parameter & ITER & \multicolumn{2}{c}{ IPP NNBI RF-Source } \\
\cline { 2 - 4 } & Requirements & BATMAN & MANITU \\
\cline { 2 - 4 } Extraction Area & $0.2 \mathrm{~m}^{2}$ & $7.0 \times 10^{-3} \mathrm{~m}^{2}$ & $1.88 \times 10^{-2} \mathrm{~m}^{2}$ \\
Calorimetric Current & $200 \mathrm{~A}^{2} \mathrm{~m}^{-}$ & $230 \mathrm{~A} / \mathrm{m}^{2} \mathrm{D}^{-}$ & $120 \mathrm{~A}^{2} \mathrm{~m}^{-}$ \\
Density & $280 \mathrm{~A} / \mathrm{m}^{2} \mathrm{H}^{-}$ & $330 \mathrm{~A} / \mathrm{m}^{2} \mathrm{H}^{-}$ & $150 \mathrm{~A} / \mathrm{m}^{2} \mathrm{H}^{-}$ \\
Extraction Voltage & $9 \mathrm{kV}$ & $9 \mathrm{kV}$ & $8 \mathrm{kV}$ (Hydrogen) \\
& & & $7 \mathrm{kV}$ (Deuterium) \\
Source Pressure & $0.3 \mathrm{~Pa}$ & $0.3 \mathrm{~Pa}$ & $0.3-0.4 \mathrm{~Pa}$ \\
Electron Content & 1 & $<1$ & $<1$ (Hydrogen) \\
(je/jH-) & & & $1.5-2$ (Deuterium) \\
Pulse Length & $3600 \mathrm{~s}$ & $<4 \mathrm{~s}$ & $<600 \mathrm{~s}$ (Hydrogen) \\
& & & $<200 \mathrm{~s}$ (Deuterium) \\
Source Dimension & $1.5 \times 0.6 \mathrm{~m}^{2}$ & $0.32 \times 0.59 \mathrm{~m}^{2}$ & $0.32 \times 0.59 \mathrm{~m}^{2}$ \\
Uniformity & $\pm 10 \%$ & t.b.d. & t.b.d. \\
\hline
\end{tabular}

* estimated from the electrically measured ion current

The IPP RF source has already demonstrated current densities in excess of the ITER requirements at the specified pressure and electron/ion ratio at the test facility BATMAN [2,3], but with only small extraction area and limited pulse length (see table 1). The further development at IPP Garching concentrates now at BATMAN on reliable deuterium operation with a low amount of co-extracted electrons, on long pulse operation (at the test facility MANITU [10]) and on source size extension (at the test facility RADI $[11,12])$.

\section{Experimental Set-Up}

\subsection{The IPP RF source}

The IPP RF driven negative ion source $[13,14]$ consists of three parts: the so-called driver, where the $\mathrm{RF}$ is coupled to the plasma, the expansion region, where the plasma expands into the actual source body, and the extraction region. The latter two are separated by a magnetic field of the order of $10 \mathrm{mT}$, the filter field. The driver is mounted on the back of the source body and consists of an alumina cylinder with a water-cooled RF coil connected to a $1 \mathrm{MHz}$ oscillator. An internal water-cooled copper Faraday screen protects the alumina cylinder from the plasma.

The subdivision of the source is necessary in order to keep the 'hot' electrons, which are generated by the RF and have energies of about $8 \mathrm{eV}$, away from the extraction region. Here electron temperatures below $2-3 \mathrm{eV}$ are necessary for minimizing the destruction rate of the negative hydrogen ions by electron collisions; then mutual neutralization with positive ions takes over as the dominant destruction process.

The extraction system is an adaptation of the IPP grid system for positive ion acceleration due to costs; the diameter of the plasma grid aperture is $8 \mathrm{~mm}$ compared to $14 \mathrm{~mm}$ in the ITER reference design. However, from the comparison with results obtained from a small ITER-like grid no major difference in the performance was seen [2].

For optimum performance, i.e. high negative ion current density and low electron current, Cs evaporation into the source is mandatory [2]. The underlying process is the "surface process", i.e. the interaction of atoms or ions with materials of low work function. Due to the short survival length of the negative ions in the plasma [15], only ions created at the plasma grid can be extracted. The negative ions produced at the plasma grid are then accelerated into the plasma by the sheath potential. First 3D trajectory calculations including all magnetic fields indicate that then the ions are back to the extraction 


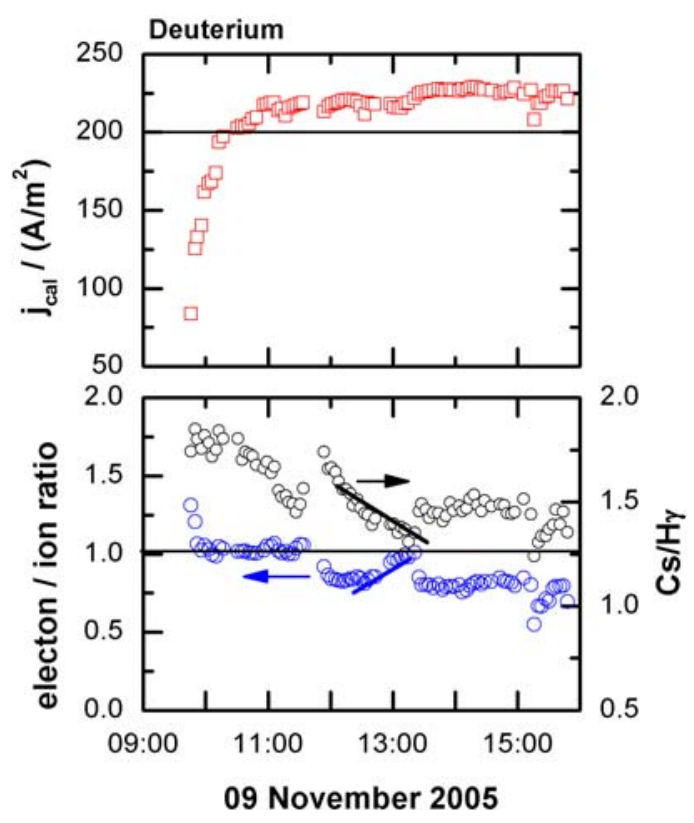

FIGURE 1: Typical daily evolution of the calorimetric current density (top) and the electron to ion ratio (bottom) at BATMAN for a well cesiated source in November 2005 for a source pressure of $0.3 \mathrm{~Pa}$ and a maximum RF power of $85 \mathrm{~kW}$. Also shown is the line ratio of the CsI emission at 852 $\mathrm{nm}$ and the $\mathrm{H} \gamma$ emission at $434 \mathrm{~nm}$. system mainly by charge exchange and the magnetic fields near the apertures [16]. The cesium coverage of the plasma grid is controlled (somewhat) by heating the plasma grid - to temperatures in the range of $100-200{ }^{\circ} \mathrm{C}-$ and by the source body temperature [2]. A further reduction of the amount of co-extracted electrons is achieved by biasing the plasma grid with $10-20 \mathrm{~V}$ against the source body together with a sufficient filter field across the plasma grid [2].

\subsection{Test Facilities}

IPP has now three test facilities in operation for the negative ion source development: (1) the small, flexible and well diagnosed test bed BATMAN (Bavarian Test Machine for Negative Ions), (2) the long pulse test facility MANITU (Multi Ampere Negative Ion Test Unit) and (3) the large source test facility RADI (re-use of the former radial injector of W7-AS) which went into operation recently.

BATMAN and MANITU are equipped with the same source; the source body dimensions $(32 \mathrm{x}$ $59 \mathrm{~cm}^{2}$ ) of this source are those of the positive ion system presently installed at ASDEX Upgrade [5]. The pulse length at BATMAN is limited to less than $4 \mathrm{~s}$ due to limitations of pumping capacity and power supplies. Due to the low total accelerated current, practically unlimited deuterium operation with regard to radiation protection is possible at BATMAN. The long pulse test facility MANITU was further upgraded to cw operation in the last year, allowing now stable operation with a pulse length of some $100 \mathrm{~s}$.

The third test facility RADI is now commissioned with first plasma pulses. RADI is equipped with the so-called half-size source with the width and half the height of the ITER source. Its aim is to demonstrate the required plasma homogeneity of a large RF source; its modular driver concept will allow an extrapolation to the full size ITER source. The details of the half-size source, the test facility and the RF circuit as well as the status of the plasma commissioning are discussed in Refs. [11] and [12]. The source is equipped with four standard IPP drivers powered by 2 RF generators. Full size extraction is presently not possible due to the lack of an insulator, a large size extraction system and a beam dump, but foreseen in a later phase (see below). The RF power supply consists of two $180 \mathrm{~kW}, 1 \mathrm{MHz} \mathrm{RF}$ generators rated for a pulse length of up to $30 \mathrm{~s}$.

MANITU and BATMAN have specially designed calorimeters for the measurement of the extracted negative ion current density. The electrical currents flowing onto the grids as well as the current flowing back to the HV power supply are measured individually. Although some ions may hit the extraction grid and some electrons may hit the grounded grid - and also secondary electrons released from the extraction grid - , extraction experiments with He that does not form negative ions indicate, that the extraction grid current refers totally to the co-extracted electrons, and the current flowing back from ground potential (grounded grid, tank, scrapers, calorimeter) is solely due to the extracted negative ions [2]. The current accountability is generally good: about $90 \%$ of the ion current can be found on the grounded grid and the calorimeter. 


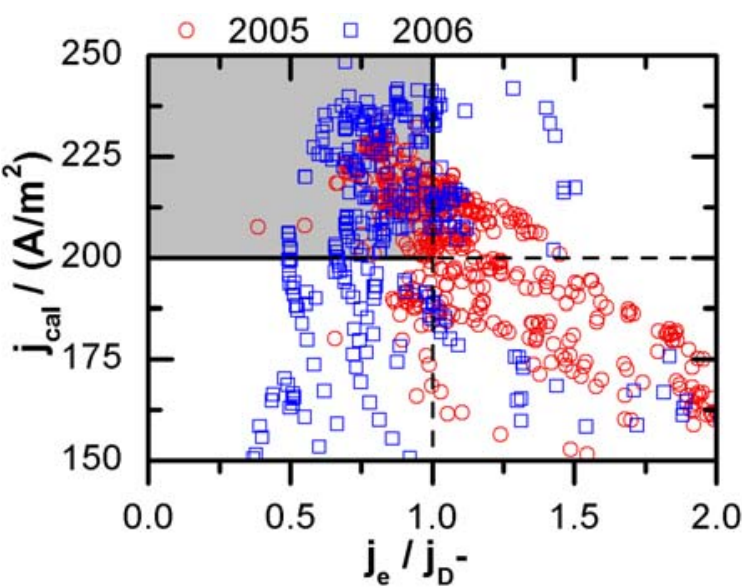

FIGURE 2: Performance of the RF source for deuterium pulses in the required pressure range in 2005 and 2006. The RF power was in the range of 80 to $90 \mathrm{~kW}$, the extraction voltage in the range of $9-10 \mathrm{kV}$, the plasma grid temperature in the range of 110 to $150^{\circ} \mathrm{C}$.

\section{Results}

\subsection{Deuterium operation}

The RF source works more efficiently with deuterium than with hydrogen [2]. This is consistent with results from optical emission spectroscopy; in deuterium discharges, the electron temperature is lower, which lowers the destruction rate due to electron collision, and furthermore the degree of dissociation is higher, which enhances the neutral flux to the surface, hence increasing the surface production of negative ions.

Problematic is the higher co-extracted electron fraction in deuterium. This higher co-extracted electron fraction, together with the higher extraction voltage required for deuterium causes problems with the power loading on the extraction grid and has limited the maximum $\mathrm{D}^{-}$current density. Hence, deuterium operation requires a larger filter field for suppressing the electron current than operation in hydrogen. As the increase of the filter field by external magnets has the drawback of a far-reaching magnetic field into the source, the local filter field across the grid was increased by a change of the filter field magnet geometry in the diagnostic flange.

Figure 1 shows a typical daily evolution of the source performance for a well cesiated source. After a few pulses the current density exceeds the required value of $200 \mathrm{~A} / \mathrm{m}^{2}$ and remains well above this number for the whole day. The small changes are due to changes in the parameters (RF power, extraction voltage, bias, and the arrangement of the confinement magnets). As changes of the parameters also change the Cs dynamics in the source, it is not obvious whether the response of the source performance is due to the parameter change or due to a change of the Cs conditioning of the source or the plasma grid.

The electron to ion ratio stays around one in the initial phase and was decreased to about 0.8 by the a change of the confinement magnet arrangement. At this time, the Cs evaporation rate was rather low, about $1 \mathrm{mg} / \mathrm{h}$. At around 1 p.m., the Cs condition of the source

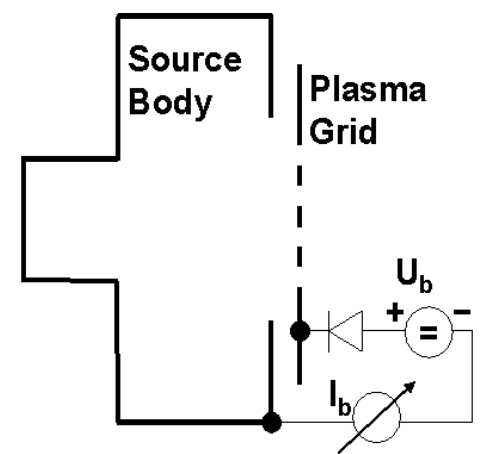

FIGURE 3: Bias circuit of the IPP RF source. The plasma grid is positively biased versus the source body; a diode prevents a change of current polarity. 'decreases', as indicated by the decrease of the line ratio of the CsI emission at $852 \mathrm{~nm}$ and the $\mathrm{H}_{\gamma}$ emission at $434 \mathrm{~nm}$ and the increase of the electron to ion ratio. This ratio is a normalized measure of the Cs density near the grid which amounts to about $1 \%$ of the electron density - the latter being in the range of $10^{17} \mathrm{~m}^{-3}$. The Cs evaporation rate was then increased by a factor of 2 . Corresponding to the increase of the $\mathrm{Cs}$ density, the electron to ion ratio was immediately reduced to 0.8 . A further reduction to about 0.5 was obtained by reducing the RF power from the initial $75 \mathrm{~kW}$ to $60 \mathrm{~kW}$ (at about 3 p.m.). This reduction also reduces the calorimetric current density, but the reduction was only small.

Figure 2 summarizes the deuterium performance of the IPP RF source at BATMAN. In total, 179 pulses have been obtained end of 2005 and in the beginning of 2006 with calorimetric current densities well above $200 \mathrm{~A} / \mathrm{m}^{2}$ and an electron to ion ratio well below one. The number was limited only by operational reasons. After an 


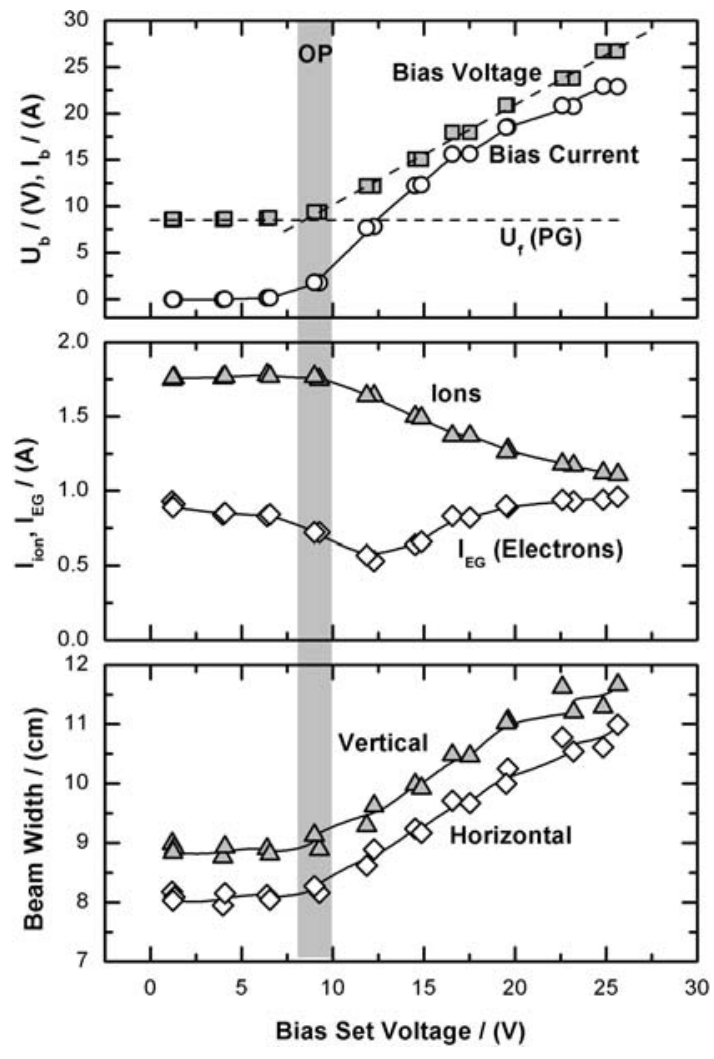

FIGURE 4: Dependence of the bias voltage $\left(\mathrm{U}_{\mathrm{b}}\right)$, bias current $\left(\mathrm{I}_{\mathrm{b}}\right)$, ion $\left(\mathrm{I}_{\mathrm{ion}}\right)$ and electron current $\left(\mathrm{I}_{\mathrm{EG}}=\right.$ current on the extraction grid) on the set bias voltage for hydrogen discharges at BATMAN. RF power was $85 \mathrm{~kW}$ and the filling pressure $0.4 \mathrm{~Pa}$. The typical operation point (OP) is the point where the bias current is just above zero and the bias voltage just above the plasma grid floating potential $\left[\mathrm{U}_{\mathrm{f}}(\mathrm{PG})\right]$.

current is obtained for a bias current just above zero, i.e. when the bias voltage is near the floating potential potential of the plasma grid. Increasing the bias current increases the coextracted electron current and reduces the ion current. In addition, it was possible to obtain a more reliable operation by controlling the bias current instead of the bias voltage.

This operational szenario is demonstrated in figures 3 and 4. Figure 3 shows the electrical bias circuit of the IPP RF sources. The plasma grid is positively biased against the source body. A diode prevents a change of the current direction in order to prevent the bias power supply from damages. A consequence of that diode is that if the bias set voltage is too small, i.e. no current flows in the circuit, the plasma grid is floating so that the current remains zero. This can be seen in figure 4 where the typical dependence of the varius currents in the source (bias, ions and electrons, respectively) on the bias set voltage is shown. Above a certain voltage, the measured bias voltage increases linearly with the set voltage and the bias current increases correspondingly. However, if the bias set voltage is too low, no current can flow, and the measured bias voltage remains constant at the floating potential of the plasma grid. As a result of the experimental expierence, as mentioned above, a stable operation of the source at high performance was obtained when the bias current was just above zero (in the case of BATMAN in the range of $1-2 \mathrm{~A}$, indicated by OP in figure 4) and the bias power supply was operated in a current control mode. As can be seen, at this operational point, the ion current is still high and the electron current is already reduced. The control of the current instead of the voltage might then ensure that the fluxes of electrons and ions to the plasma grid are constant during the discharge 
$0.3 \mathrm{~Pa}, 70 \mathrm{~kW}$

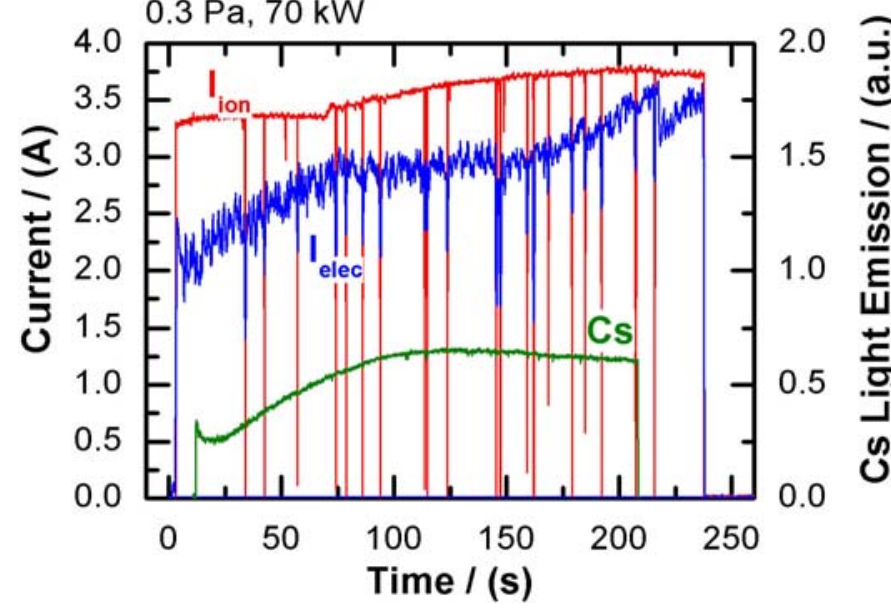

FIGURE 5: Time traces of a 240 second pulse at MANITU. $\mathrm{I}_{\text {ion }}$ denotes the electrically measured ion current, and $\mathrm{I}_{\text {elec }}$ the electron current on the extraction grid. The 'decay' of the Cs signal at 210 seconds is due to a switch-off of the spectrometer. The maximum ion current of 3.8 A corresponds to an electrically measured current density of $202 \mathrm{~A} / \mathrm{m}^{2}$ and an estimated calorimetric current density of $150-160 \mathrm{~A} / \mathrm{m}^{2}$. The pressure was in the range of $0.3-0.4 \mathrm{~Pa}$, the RF power $70 \mathrm{~kW}$. and hence stabilize the performance of the source. The increase of the current on the extraction grid with increasing bias set voltage above about $12 \mathrm{~V}$ might be explained by a increased loss also of negative ions on that grid; this is supported by the corresponding increase of the beam width.

\subsection{Long Pulse Experiments}

The long pulse test facility MANITU was further upgraded to $\mathrm{cw}$ operation in the last year by installing an actively cooled Faraday screen and an air cooled plasma grid, by an improved cooling of the back plate of the source and by temperature control of the source body. The radiation protection for deuterium operation consists of a $30 \mathrm{~cm}$ thick water tank around the calorimeter and a $20 \mathrm{~cm}$ thick polyethylene wall around the source allowing $6 \mathrm{~h}$ operation per year.

The air cooled plasma grid is derived from a plasma grid of the AUG positive ion system, which has cooling channels between each row of extraction holes, but with a $3 \mathrm{~mm}$ deep chamfer on the plasma side. The extraction area is reduced to a size suitable for negative ions by a molybdenum mask, at present $0.0188 \mathrm{~m}^{2}$. This mask is attached to the plasma grid without a cooling loop of its own. Using the mask has the advantage that the largest possible extraction area can easily be identified by varying the opening in the mask; however, the mask will heat up during long pulses and thus limit the pulse length to about 10 minutes.

A typical example of a long pulse at MANITU in hydrogen is shown in Figure 5. The ion current remains constant for the first $70 \mathrm{~s}$ of beam time and an increase to about $3.8 \mathrm{~A}\left(202 \mathrm{~A} / \mathrm{m}^{2}\right)$ at the end of the pulse. As mentioned above, the beam calorimetry is not reliable, but from the former short pulse experiments at MANITU [3], [10], where the extracted beam current was measured with an inertially cooled calorimeter, a calorimetric current of about $150 \mathrm{~A} / \mathrm{m}^{2}$ can be estimated. Best results obtained so far in hydrogen have been ion currents approaching $4 \mathrm{~A}\left(210 \mathrm{~A} / \mathrm{m}^{2}\right)$ and pulses of up to $600 \mathrm{~s}$. Common to all pulses in excess of $200 \mathrm{~s}$ is a rising electron current with time, which is accompanied by a rising intensity of the Cs852 line. This is most probably a consequence of the un-cooled grid cover and limits the pulse length in deuterium to about $200 \mathrm{~s}$.

So far there is no indication that cesium consumption is affected by the pulse duration. During the conditioning phase typically a total of $50-100 \mathrm{mg}$ of cesium continuously over $6-8$ hours was evaporated into the source. Within one month this accumulated to about $500 \mathrm{mg}$. However, the loss of cesium due to the vapor pressure is considerably smaller than expected from the vapor pressure of bulk cesium and the grid conductance. A reason for this low loss rate might be that the vapor pressure of thin layers on a metal surface is much smaller than for bulk material.

MANITU is equipped with a spatially resolved beam emission spectroscopy system [17] for measurements of the beam homogeneity and their dependence on the extraction area. The temporal and spatial homogeneity of the ion beam can be determined by comparing the half width of the Doppler shifted $\mathrm{H}_{\alpha}$ line. First preliminary measurements showed that the beam inhomogeneity is less than 5 - 


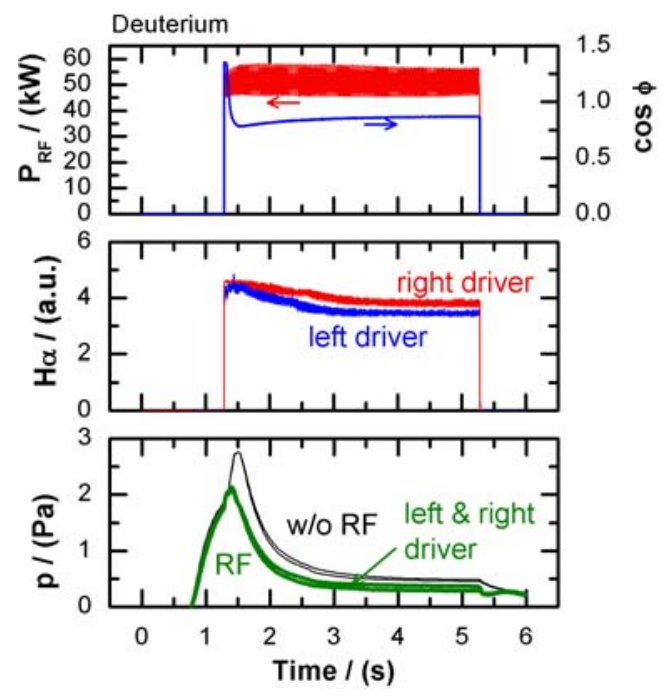

FIGURE 6: First example of a $50 \mathrm{~kW}$ discharge at RADI in deuterium. Only the two lower drivers are powered in series with one generator. The pressure in the drivers with plasma is reduced during the RF phase compared to the drivers w/o plasma. A gas puff is necessary for plasma ignition.

modular driver concept will allow an extrapolation to the full size ITER source without any change of the source width.

The half-size source is devoted to demonstrate the required homogeneity of large RF plasmas, to investigate the Cs dynamics in a large RF source as well as to test the geometry and the optimum number of drivers and to test an ITER-like RF circuit. The experience of the operation of this source will then be used in the final design of the ITER RF source that was started in the last year by RFX Padua in collaboration with IPP Garching. The test facility has presently been commissioned with the first plasma pulses (see figure 6).

The half-size source design offers a large amount of flexibility regarding the magnetic configuration, the source depth, the gas flow and the driver geometry. The source depth can be changed - when the source is vented - by a variable back plate that hosts the drivers. A dummy grid with adjustable slits allows matching the conductance of the ITER source. As extraction is presently not foreseen, the generator and the source are at the same potential, similar to the RF circuit that is foreseen for the ITER NNBI system. The filter field can be generated like in the ITER source by a current flowing through the plasma grid in combination with permanent magnets.

As large area extraction is not yet foreseen presently, measurements of the extracted ion and electron current densities are not possible. Hence, IPP Garching is currently investigating and testing plasma diagnostic tools (optical emission spectroscopy [12,15,18,19], Langmuir probes [20], Laser detachment [20] and cavity ringdown spectroscopy [21,22]). These tools have been successfully commissioned in an RF and HV environment at BATMAN (and MANITU). An example is shown in figure 7.

\section{Future Plans}

The experiments on BATMAN concentrate now on the physical understanding of the cesium dynamics and on the production and extraction of the negative ions as well as on deuterium operation with a low amount of co-extracted electrons. The detailed processes of negative ion formation and destruc- 


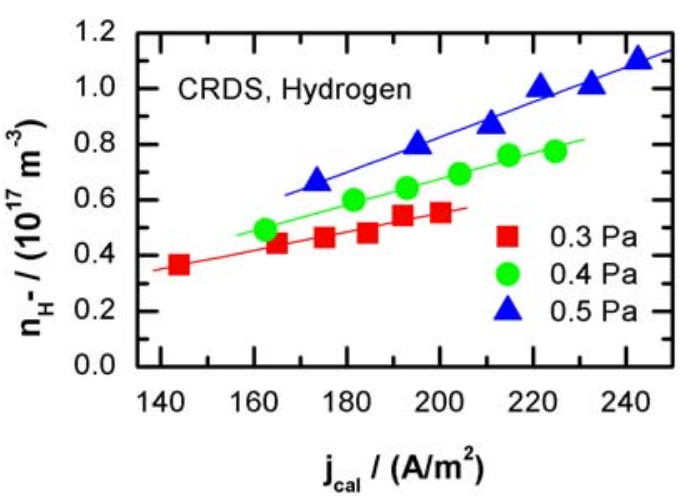

FIGURE 7: Correlation of the negative ion density in front of the plasma grid with the extracted ion current density as measured by Cavity ringdown spectroscopy at BATMAN [21]. he dummy plasma grid. The homogeneity can be changed by different power levels of the drivers. The experiments in the next months concentrate on the influence of the magnetic filter field on the homogeneity together with biasing the plasma grid.

Although RADI will demonstrate the operation of a large RF source and the homogeneity of large RF plasmas, the results will have limited significance. The extraction, i.e. the potential distribution within the plasma, changes the plasma parameters in front of the plasma grid. Hence, extraction plays an important role not only for the negative ion and electron transport, but also for the Cs dynamics. Furthermore, there is evidence, that the magnetic filter field which is required for the suppression of the co-extracted electrons causes the spatial plasma and beam non-uniformity as it is observed in large filamented ion sources. It is therefore essential to optimise the magnetic filter field (and the plasma grid bias) with respect to the plasma uniformity together with a sufficiently suppressed co-extracted electron current.

Hence, long pulse large scale extraction from the $1 / 2$-size ITER source is highly desirable for a better understanding of the physics and the performance of a large RF source. To fully exploit this experiment, at least the plasma source has to be operated with long pulse, as the consumption and redistribution of the cesium during a pulse is regarded as the main factor possibly limiting the long pulse performance. While the plasma source has to run continuously for an extended time it is acceptable to extract a beam - using the existing IPP HV power supply system with a duty cycle of $10 \mathrm{~s} / 160 \mathrm{~s}-$ repetitively throughout the plasma pulse.

The new project will replace the RADI test facility and (to a large extent) makes use of existing hardware at IPP. First design studies for a " $1 / 2$-size" long pulse extraction system on the basis of the SINGAP design [23] with three stages and rated for $20 \mathrm{~A}$ extracted current at $60 \mathrm{kV}$ have been started. The experience of the manufacturing and operation of such a large scale, long pulse extraction system may have some influence on the design and the construction of the ITER NNBI system.

\section{Summary}

The IPP RF source has demonstrated its principal suitability for the ITER neutral beam system. The required values in deuterium operation of current density and electron/ion ratio at the required source pressure of $0.3 \mathrm{~Pa}$ have been exceeded for short pulses $(<4 \mathrm{~s})$ and small extraction area $\left(7 \times 10^{-3} \mathrm{~m}^{2}\right)$. Reliable and reproducible deuterium operation was obtained by monitoring the amount of $\mathrm{Cs}$ in the source by optical emission spectroscopy as well as controlling the temperature of source and plasma grid, with an optimum range of about $40{ }^{\circ} \mathrm{C}$ and $100-150{ }^{\circ} \mathrm{C}$, respectively. 
The further development concentrates now on extending the pulse length and the source size. Stable pulses with a pulse length of several 100 seconds have been already achieved in hydrogen with an electron/ion below 1 and current densities of about $150 \mathrm{~A} / \mathrm{m}^{2}$. Operation in deuterium has just started.

The large source test facility has presently been commissioned with first plasma pulses; the installed 'half-size' source - with the width and half the height of the ITER source - is devoted to demonstrate the required plasma uniformity as well as to test ITER-like RF circuits. First promising results showed a sufficient homogeneity of a pure RF plasma and a sufficient overlap of the plasma near the plasma grid.

\section{References}

[1] "ITER Technical Basis", ITER EDA Documentation Series No. 24, Plant Description Document, Sec. 2.5.1. International Atomic Energy Agency (IAEA), 2002

[2] E. SPETH, Nuclear Fusion 46(6) 2006, S220

[3] P. FRANZEN et al. Fusion Engineering and Design 74 (2004) 351

[4] E. SPETH et al. Proceedings of the 20th Symposium on Fusion Technology, Marseille, 1998. Association EURATOM-CEA, Saint Paul Lez Durance, 1998, 27.

[5] O. VOLLMER et al. Proceedings of the 20th Symposium on Fusion Technology, Marseille, 1998. Association EURATOM-CEA, Saint Paul Lez Durance, 1998, 449

[6] E. SPETH, NBI Team. Plasma Science and Technology 6, 2004, 2135

[7] A. KRYLOV et al. Nuclear Fusion 46 (2006) S324

[8] K. IKEDA et al. Review of Scientific Instruments 75(5), 2004, 1744

[9] N. UMEDA Nucl. Fusion 43 (2003) 522-526

[10] W. KRAUS et al. Fusion Engineering and Design 74 (2004) 337

[11] M. FRÖSCHLE et al, this conference

[12] P. FRANZEN. To be published

[13] W. KRAUS et al. Fusion Engineering and Design, 56-57 (2001) 499

[14] O. VOLLMER et al. Fusion Engineering and Design, 56-57 (2001) 465

[15] U. FANTZ et al. Nuclear Fusion 46 (2006) S297

[16] D. WÜNDERLICH. Proc. PNNIB Conf. Sept. 2006, Santa Fe, US

[17] A. LORENZ, U. Fantz, P. Franzen. Technical Report IPP 4/285, 2006

[18] U. FANTZ et al. Fusion Engineering and Design 74 (2004) 299

[19] U. FANTZ, D. Wünderlich. New Journal of Physics, 8, 2006, 301

[20] S. CHRIST, U. Fantz. To be published

[21] M. BERGER. PhD Thesis, University of Augsburg, 2006

[22] M. BERGER, U. Fantz. To be published

[23] L. SVENSSON et al. Nuclear Fusion 46 (2006) S369 